\title{
Void-creating Algorithm in OPS/OBS: Mind the Gap
}

\author{
Kurt Van Hautegem, Wouter Rogiest and Herwig Bruneel \\ SMACS Research Group \\ Department of Telecommunications and Information Processing (TELIN); Ghent University \\ St.-Pietersnieuwstraat 41; B-9000 Ghent, Belgium \\ Email: $\{$ kurt.vanhautegem $\bowtie$, wouter.rogiest, hb\}@telin.ugent.be
}

\begin{abstract}
In optical packet/burst switching, packet contention on a single wavelength is resolved effectively by means of Fiber Delay Lines. The involved scheduling algorithm is typically designed to minimize packet loss, by filling so-called voids, and trying to keep the so-called gaps small. We propose a new type of algorithm that selectively creates voids that are larger than strictly needed, only when these will likely be filled. Results obtained by Monte Carlo simulation show that selective void creation can significantly reduce packet loss.
\end{abstract}

\section{INTRODUCTION}

Promising solutions to address the rising demand in bandwidth are the packet-based switching techniques optical packet/burst switching (OPS/OBS) in which network links can be shared among communication sessions, thereby increasing the usage of the available fiber capacity. In packet-based switching contention may arise in the network nodes when more than one packet heads for the same output port at the same time. As a solution temporary buffering is currently implemented with Fiber Delay Lines (FDLs) [1] in which the optical signals are sent through long pieces of coiled fiber to delay them for a certain time. As the number of FDLs is limited, it is necessary to schedule arriving packets as efficiently as possible in order to reduce the probability of unresolved contention, and thus packet loss.

In [2] and [3] we proposed new cost-based scheduling algorithms, both in a related setting with wavelength converters present. In both, we were able to validate the usefulness of a cost-based approach and achieved an increased performance (decreased loss probability (LP)). Although these algorithms can improve the performance significantly, their structure is very similar to existing algorithms. Indeed, they can also be split up in two big categories: void-filling and non-void-filling algorithms. In contrast to the latter, the former allow packets to be scheduled before already scheduled packets, in this way increasing performance but also the computational complexity by keeping track of voids which are not likely to be filled. We therefore propose a new type of algorithm that selectively creates larger voids, creating larger voids only when they will likely be filled. This type of algorithm does not only fill the available voids, it also controls the creation of the voids, making it more powerful, enabling better switch performance.

\section{ASSUMPTIONS}

Throughout the paper a continuous-time setting is supposed. We consider a single output port of the switch, at which packets arrive on a single wavelength $\lambda_{1}$ according to a Poisson process, with exponentially distributed inter-arrival times $T$ and an average $E[T]$. The length of the arriving packets is assumed a fixed length $B$. The nature of this arrival process implies possible overlap of distinct packets at the input causing contention which has to be resolved before the packets exit the output port, also on the single wavelength $\lambda_{1}$.

We assume no wavelength converters are present at the output port. While other wavelengths may be used for packet switching within the same switch they operate independently of each other and all packets are processed on the same wavelength upon which they arrive. The output port has an optical buffer in which $N+1$ FDLs are available to schedule incoming packets. This is shown in Fig 1 (left). The lengths of the FDLs are consecutive multiples of a basic value $D$ called the granularity which is assumed equal to the fixed packet length, $D=B$. Incoming packets sent through the $j$-th $(j=0 \ldots N)$ delay line encounter a delay of $j \cdot D$. Related, the overall traffic load is given by $\rho=B / E[T]$. 


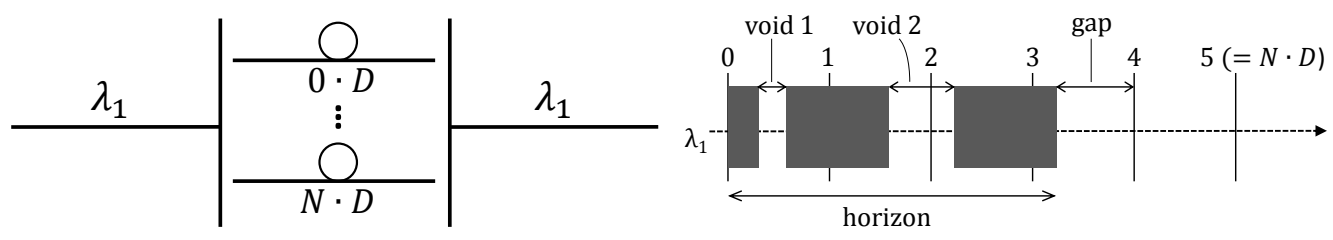

FIGURE 1. The output port with FDLs (left) and an example of a provisional schedule (right)

\section{SCHEDULING BASICS}

Scheduling is done separately for each packet upon its arrival. In this setting this amounts to the assignment of a single variable $(j)$ to the packet, corresponding to the delay line $(j=0 \ldots N)$ the packet is scheduled on. This is done by means of a provisional schedule, of which an example is given in Fig. 1 (right), showing the already scheduled packets (grey boxes) upon arrival of an arbitrary packet that is yet to be scheduled (and is thus not displayed on the provisional schedule). The provisional schedule is represented horizontally and the vertical lines represent the delays of the FDLs $(j=0 \ldots N=5)$. In this representation, the provisional schedule evolution can be seen as a choppy (observed from arrival to arrival) but uniform (all packets move alike) movement of all packets to the left, with packets disappearing (because they are being transmitted) when crossing the zero delay reference line. A packet has to be scheduled on an FDL without overlapping with any of the already present packets. If no such FDL is available, the packet is lost.

Scheduling is done according to a scheduling algorithm, of which most are designed purely for minimal loss. Regardless of their exact design aim, existing scheduling algorithms can be split up in two main categories:

- void-filling algorithms allow packets to be scheduled on any suitable FDL. This implies the possibility of filling up voids, defined as unscheduled periods followed by one or more packet scheduled beyond it. Here, only voids overlapping an FDL more than a packet length to the right of the FDL are fillable.

- non-void-filling algorithms only allow packets to join at the back and voids can not be filled. In this way the algorithm is not obliged to keep track of all voids but merely of the horizon, defined as the latest time at which the output is currently scheduled to be in use. Graphically, this corresponds to the right edge of the rightmost packet. Related the gap is defined as the length of the void in front of a packet when it is assigned to the first FDL to the right of the horizon. Or alternatively: gap $=\left\lceil\frac{\text { horizon }}{D}\right\rceil \cdot D-$ horizon.

As $D=B$ in the example of Fig. 1 (right) no voids equal or larger than the packet length (fillable voids) will be created and thus none can be filled. Only voids smaller than the packet length (unfillable voids) are created. In this setting of a single wavelength, and $D=B$, all void-filling and non-void-filling algorithms will schedule the packet on the first FDL to the right of the horizon. This is unless the horizon is larger than $N \cdot D$, in which case the packet is lost.

\section{VOID-CREATING SCHEDULING ALGORITHM}

Minimizing the gaps positively impacts the number of packets lost. As small gaps make the stacking of the packets dense, it reduces the chance the horizon exceeds $N \cdot D$ in which case a packet is lost. When a fillable void created by a first packet can be filled by any subsequent packet the average gap encountered per packet will typically be lower than in the case a fillable void was never created. On the other hand creating but not filling voids is disadvantageous for the performance as the stacking becomes less dense, and the chance the horizons exceeds $N \cdot D$ increases.

In order to improve the performance of this system, fillable voids are created by scheduling the packet on the second instead of the first FDL to the right of the horizon. As $D=B$ this will create voids larger than $B$ (fillable voids) which, if favourably positioned with respect to an FDL, can be filled. Whether a favourable position will occur, depends on the choppy evolution of the provisional schedule and thus on the stochastic arrival process. We can however maximise the chance of filling up a void by creating only voids that are likely to be filled. Besides allowing only a single fillable void in the provisional schedule we demand for two conditions to be met before creating a fillable void: 

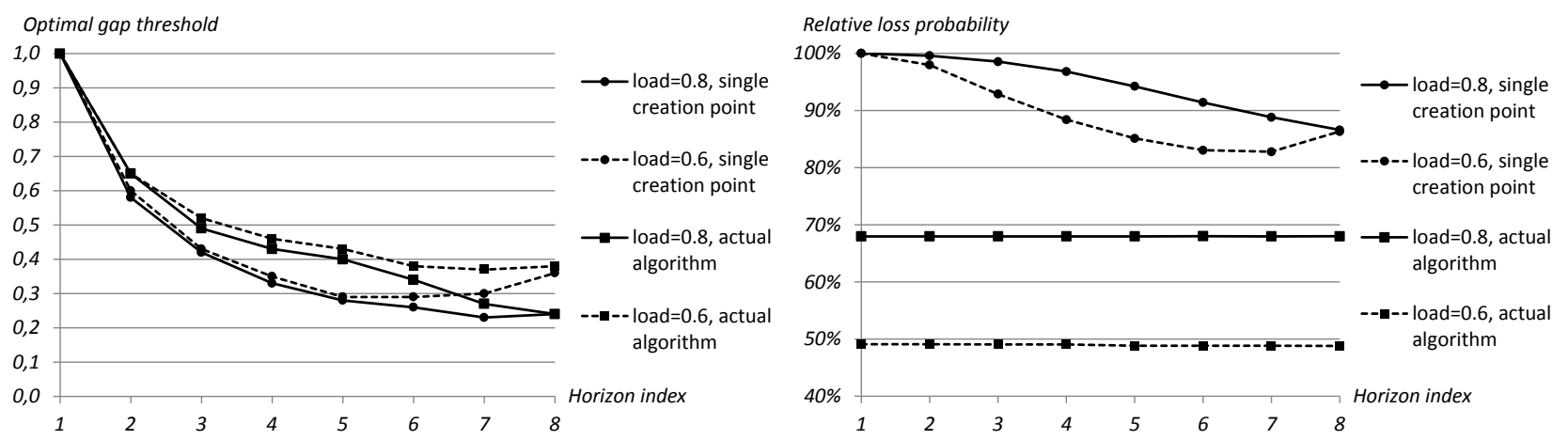

FIGURE 2. Optimal gap threshold (left) and relative loss probability (right) versus horizon index

- A first condition relates to the size of the created void. As the void-creating algorithm chooses the second FDL to the right of the horizon, the created voids will have a length between $D$ and $2 \cdot D$. A void only slightly larger than $D$ will have a small range of favourable positions with respect to the FDLs. To fill a void, it indeed has to overlap an FDL at least a length of $D=B$ to the right of the FDL on a set of future, yet unknown, arrival instances. For a void almost equal to $2 \cdot D$ it is very likely it will have a favourable position with respect to an FDL, although it is still possible an unfavourable arrival pattern occurs. The first condition therefore states that the created void has to be larger than a certain value $D+y \cdot D$, i.e. $D+y \cdot D<$ fillable void. Here $y$ is called the gap threshold and is varied in simulations $(0<y<1)$.

- A second condition states that the horizon has to be between two specific and consecutive FDLs: $(n-1) \cdot D<$ horizon $<n \cdot D$ in which $n$ is called the horizon index and is varied in simulations $(n=1 \ldots N-1)$. With this condition we first want to create auxiliary results that will make it easier to obtain the optimal gap thresholds of the actual algorithm. Moreover in this way we want to investigate the effect of the position of void creation. Fillable voids created when $n$ is closer to $N-1$ will stay longer in the system and thus given the Poisson arrival process, have a higher chance of being filled. When the horizon is an exact multiple of $D$, which for a continuous time system only occurs for an empty buffer and thus a horizon of zero, the assigned gap is zero when the packet is scheduled on the first FDL to the right of the horizon. As a gap of zero cannot be improved by creating and filling a void, this condition therefore uses the strictly larger than signs.

If one of these conditions is not met or another fillable void is already present in the provisional schedule, the packet is used to fill this void or otherwise is scheduled on the first FDL to the right of the horizon, creating an unfillable void.

\section{PERFORMANCE RESULTS}

We first simulate the system without void creation, i.e. all packets are scheduled on the first FDL to the right of the horizon. If the horizon is larger than $N \cdot D$ on arrival of a packet, it is lost. The number of FDLs was assumed ten $(N+1=10)$ and $D=B$ as stated earlier. For a load $\rho$ of $80 \%$ this gives a loss of $14.46 \%$ of the arriving packets. When the load is $60 \%$ the loss is $2.08 \%$. These values are not shown on Fig 2.

A second, auxiliary, set of simulations allows to create a fillable void when the two conditions are met, i.e. $D+y \cdot D<$ fillable void and $(n-1) \cdot D<$ horizon $<n \cdot D$. The results of these simulations are shown in Fig 2 . The curves marked as 'single creation point' in Fig 2 (left) show the optimal gap thresholds for the different horizon indexes, when void creation is only allowed for that index. For example when a fillable void is allowed to be created if (and only if) $D<$ horizon $<2 \cdot D$, then the void has to be larger than $1.6 \cdot D(y>0.6)$ for a load of $60 \%$ to achieve the largest reduction in LP. The LPs with the threshold values of Fig 2 (left) are shown in Fig 2 (right). The LPs are shown relative to the LPs when no void creation is allowed, i.e. $2.08 \%$ and $14.46 \%$ for a load of $60 \%$ and $80 \%$ respectively. The number of simulated packets is $10 \cdot 10^{6}$ for all simulations which gives confidence intervals too narrow to be displayed on the figures. 
A third set of simulations determines the optimal gap thresholds in a scenario where a fillable void is allowed to be created on all feasible positions for the horizon $(0<$ horizon $<(N-1) \cdot D)$. With these simulations we thus obtain the results for the actual algorithm. Since voids can be created at each horizon index $(n=1 \ldots N-1)$, a different optimal gap threshold needs to be determined for each. As the parameter set over which has to be optimised is too large to simulate in an acceptable time, these values are approximated by an iterative method. In a first iteration we fixed all eight gap thresholds but one to their optimal value from the 'single creation point' graphs. The one that is not fixed is optimised, and this is done for all eight positions. This gave us a set of eight new optimal gap thresholds, one for each horizon index. In a second iteration we fixed all gap thresholds but one to these newly obtained values and again optimised the one that was not fixed. Finding little difference with the first iteration, the results of the second iteration are acceptable as good approximation of the optimal values. The optimal values of the gap thresholds and relative LPs obtained with this second iteration are shown in Fig 2 on the graphs marked as 'actual algorithm'.

We can see that for single creation point the lower load allows for a bigger relative reduction in LP. For equal horizon index, the optimal gap threshold is larger, and thus stricter, for the lower load. This is because for lower load the number of expected packets to arrive, before the void disappears by crossing the zero delay line, is lower. This lack of high arrival density thus has to be compensated by a larger size of the created void. For both load values no improvement in LP is achieved when the fillable void is allowed to be created when $n=1$. This is indicated by an optimal gap threshold of 1 for $n=1$, as this never occurs. For a load of $80 \%$ the optimal LP monotonically decreases with an increasing horizon index. Opposed to this, for a load of $60 \%$ less improvement in LP is possible when fillable voids are created when $n=8$ than when $n=7$. A possible explanation is that when the load is 'low' (60\%), the probability of finding a horizon index $n=8$ is smaller than for higher load $(80 \%)$, thus reducing the number of instances fillable voids can be created and the LP is reduced. The load for which this inversion is observed may be linked to the critical load of $69.3 \%$ for which the infinite system without void creation becomes unstable $(\rho=\ln (2)$, see[4]).

As the 'actual algorithm' curves illustrate, a lower load also allows for a bigger reduction in LP. For a load of $60 \%$ there is reduction of more than $50 \%$ in LP. This reduction is practically the same for all values of $n$ as for all these simulation points void creation is allowed for all positions of the horizon, each with their distinct gap threshold. The optimal gap thresholds are higher, and thus stricter, than for the corresponding 'single creation point' graphs. As void creation is also allowed for other positions of the horizon, a stricter policy can indeed be applied.

\section{CONCLUSIONS}

In this paper we proposed a new type of algorithm called void creating algorithm. Using Monte Carlo simulation this algorithm was validated for a single-wavelength fixed packet length setting. It was shown that by selectively creating larger voids than strictly necessary, this algorithm can achieve performance improvements of up to $30 \%$ for a high load $(80 \%)$ and $50 \%$ for a lower load $(60 \%)$. These results open opportunities to analyse the algorithm in more complex settings as well as analysing its mechanism in a more mathematical way.

\section{ACKNOWLEDGMENTS}

Part of this research was funded by the Interuniversity Attraction Poles Programme initiated by the Belgian Science Policy Office. The second author is Postdoctoral Fellow with the Research Foundation Flanders (FWO-Vlaanderen).

\section{REFERENCES}

1. E. Burmeister, D. Blumenthal, and J. Bowers, Optical Switching and Networking 5, 10 - 18 (2008), ISSN 1573-4277.

2. K. Van Hautegem, W. Rogiest, and H. Bruneel, "OPS/OBS Scheduling Algorithms: Incorporating a Wavelength Conversion Cost in the Performance Analysis," in Proceedings of the 32nd IEEE International Performance, Computing, and Communication Conference (IPCCC), San Diego,California,USA, 2013.

3. K. Van Hautegem, W. Rogiest, and H. Bruneel, "Scheduling in Optical Switching: Deploying Shared Wavelength Converters More Effectively," in Proceedings of the 2014 IEEE International Conference on Communications (ICC), Sydney, 2014.

4. W. Rogiest, J. Lambert, D. Fiems, B. V. Houdt, H. Bruneel, and C. Blondia, Performance Evaluation 66, 343 - 355 (2009). 